\title{
Seroprevalencia del virus del Síndrome Reproductivo y Respiratorio Porcino en cerdos de crianza no tecnificada del Perú
}

\author{
Seroprevalence of the Porcine Reproductive and Respiratory Syndrome \\ VIRUS IN NON-TECHNICALLY MANAGED PIGS HERDS OF PERU
}

\author{
Miguel Quevedo V. ${ }^{1,3}$, Jorge Mantilla S. ${ }^{1}$, Katherine Portilla J. ${ }^{1}$, \\ Rubén Villacaqui A. ${ }^{1}$, Hermelinda Rivera G. $^{2}$
}

\section{Resumen}

El objetivo del presente estudio fue determinar la seroprevalencia del virus del Síndrome Reproductivo y Respiratorio Porcino (VPRRS) en cerdos de crianzas no tecnificadas de 23 de los 24 departamentos del Perú. Se colectaron 4526 muestras de sangre de cerdos machos y hembras mayores de cuatro semanas entre octubre de 2015 y marzo de 2016 para la detección de anticuerpos contra el VPRRS mediante la prueba de ELISA indirecta. El 17.3\% ( $\left.\mathrm{IC}_{95 \%}: 16.3-18.5\right)$ de los cerdos tuvieron anticuerpos contra el VPRRS. El promedio de la seroprevalencia del virus fue de $25.7 \%\left(\mathrm{IC}_{95 \%}: 23.6-27.9\right)$ en los cerdos de la zona centro, seguido por los de la zona norte con $14.8 \%\left(\mathrm{IC}_{95 \%}: 12.9-16.7\right)$ y zona sur del país con $11.5 \%\left(\mathrm{IC}_{95 \%}: 10.0-13.1\right)$. La seroprevalencia del VPRRS en los cerdos del departamento de Lima fue de $62.2 \%\left(\mathrm{IC}_{95 \%}: 57.7-66.6\right)$, seguido por cerdos del departamento de Arequipa con $37.9 \%\left(\mathrm{IC}_{95 \%}: 31.1-44.6\right)$ y Lambayeque con $31.3 \%\left(\mathrm{IC}_{95 \%}: 22.0-40.5\right)$. Hubo asociación estadística $(\mathrm{p}<0.05)$ entre el sexo de los cerdos y la presencia de anticuerpos contra el VPRRS dentro del grupo de animales de 27 a 52 semanas. La presencia de anticuerpos contra el VPRRS detectado en cerdos de crianza no tecnificada de todo el país indica que los animales fueron expuestos al virus de campo, ya que la prevención de la enfermedad mediante la vacunación no está permitida en el país.

Palabras clave: cerdos; VPRRS; anticuerpos; seroprevalencia; crianza no tecnificada

\footnotetext{
${ }^{1}$ Dirección de Sanidad Animal, Servicio Nacional de Sanidad Agraria, Ministerio de Agricultura y Riego, Lima, Perú

${ }^{2}$ Laboratorio de Microbiología y Parasitología Veterinaria, Facultad de Medicina Veterinaria, Universidad Nacional Mayor de San Marcos, Lima, Perú

${ }^{3}$ E-mail: mquevedom@senasa.gob.pe
}

Recibido: 18 de octubre de 2017

Aceptado para publicación: 9 de febrero de 2018 
The objective of this study was to determine the seroprevalence of the Porcine Reproductive and Respiratory Syndrome Virus (VPRRS) in non-technologically managed pig herds of 23 departments of Peru. Blood samples $(\mathrm{n}=4526)$ were collected from male and female pigs older than four weeks of age between October 2015 and March 2016 for the detection of antibodies against the VPRRS by the indirect ELISA test. The 17.3\% (95\% CI: 16.3-18.5) of the pigs had antibodies against the VPRRS. The average seroprevalence of the virus was $25.7 \%$ (95\% CI: $23.6-27.9)$ in pigs of the central region, followed by those of the northern region with $14.8 \%$ (95\% CI: 12.9-16.7) and southern area of the country with $11.5 \%$ (95\% CI: 10.0-13.1). The seroprevalence of the VPRRS in pigs of the department of Lima was $62.2 \%$ (95\% CI: 57.7-66.6), followed by pigs from the department of Arequipa with $37.9 \%(95 \% \mathrm{CI}: 31.1-44.6)$ and Lambayeque with $31.3 \%(95 \%$ CI: 22.0-40.5). There was statistical association $(p<0.05)$ between the sex of the pigs and the presence of antibodies against the VPRRS within the group of animals from 27 to 52 weeks. The presence of antibodies against the VPRRS detected in non-technologically managed farms across the country indicates that the animals were exposed to the field virus, since the prevention of the disease through vaccination is not allowed in the country.

Key words: pig; PRRSV; antibodies; prevalence; poor managed pig herds

\section{INTRODUCCIÓN}

El desarrollo de la producción porcina mundial en las últimas décadas se debe, entre otros factores, al incremento global de la demanda de proteína de origen animal para el consumo humano. Este crecimiento de la porcicultura implica la intensificación de la producción, uso de modernas tecnologías reproductivas, crianza especializada por etapas productivas, mayor movimiento de cerdos, y selección de germoplasma (Drew, 2011). Si bien el sistema de producción con alta densidad de cerdos es más eficiente, también constituye un factor predisponente al surgimiento de enfermedades, sobre todo emergentes y reemergentes, que amenazan el desarrollo de la porcicultura mundial (Holtkamp et al., 2013).

El Perú tiene una población de $2.2 \mathrm{mi}-$ 1lones de cerdos, de los cuales, el $67.2 \%$ son criollos y el $32.8 \%$ son de razas mejoradas. Asimismo, el 51.1\% de la población se en- cuentra en la sierra, donde el $89.9 \%$ son cerdos criollos (INEI, 2012) criados en forma extensiva y en forma complementaria a otras actividades agropecuarias predominantes. En la costa del país se encuentra la mayoría de granjas con modernas tecnologías y sistemas sanitarios, aunque también existen las crianzas semi-tecnificadas donde los animales poseen mejoramiento genético intermedio y un sistema sanitario básico (Morales et al., 2014).

La porcicultura nacional ha tenido un crecimiento de $1.7 \%$ (INEI, 2012), con una tendencia al desarrollo de granjas porcinas con mayor densidad de animales y con producciones más eficientes. En este contexto, el Servicio Nacional de Sanidad Agraria (SENASA) del Perú, institución oficial encargada de velar por la sanidad animal, tiene implementado un Sistema Nacional de Vigilancia Epidemiológica para enfermedades emergentes de impacto en la producción porcina como peste porcina clásica, síndrome reproductivo y respiratorio porcino, dia- 
rrea epidémica porcina y enfermedad de Aujeszky, entre otras, que vienen afectando la porcicultura mundial.

El Síndrome Reproductivo y Respiratorio Porcino (PRRS por su sigla en inglés) es una enfermedad de distribución mundial y una de las enfermedades con mayor impacto económico por afectar varios sistemas del organismo animal (OIE, 2004). Es producida por el virus del PRRS (VPRRS), perteneciente a la familia Arteriviridae. Se conocen dos genotipos, el Europeo o genotipo 1 y el Americano o genotipo 2 (Snijder et al., 2013). El VPRRS se transmite por contacto directo, de la madre al feto, a través del semen, por cerdos aparentemente sanos y por fómites (Lambert et al., 2012). Esta enfermedad se caracteriza principalmente por causar desórdenes reproductivos y respiratorios, así como predisponer a infecciones secundarias del tracto respiratorio en animales jóvenes y adultos (Zimmerman et al., 1997).

El PRRS ha sido detectado serológicamente en muestras de suero de cerdos con ligeros o sin signos respiratorios de granjas tecnificadas de Lima (Alegría et al., 1998; Calcina et al., 2014). Ramírez et al. (2013) aislaron e identificaron las cepas del VPRRS del genotipo 1 de granjas porcinas de las provincias de Lima y Arequipa, a partir de muestras de sueros de cerdos sin evidencia de signos clínicos. Análisis serológicos posteriores realizados en granjas tecnificadas de la costa del país indican que el VPRRS está ampliamente difundido; sin embargo, no se tienen estudios serológicos del VPRRS en cerdos de crianzas no tecnificadas, por lo que el objetivo del estudio fue determinar la seroprevalencia del VPRRS en cerdos de estas crianzas a nivel nacional, como base para elaborar estrategias de prevención y control del PRRS.

\section{Materiales y Métodos}

\section{Diseño y Marco Muestral}

El estudio fue de tipo descriptivo transversal, realizado de manera bietápica mediante conglomerados. Se estableció como marco muestral la diferencia de la población porcina del IV Censo Agropecuario (INEI, 2012) y la de las crianzas tecnificadas registradas en el SENASA, por lo que se consideró una población de crianza no tecnificada a 2229475 cerdos distribuidos en 23 de los 24 departamentos del Perú (el departamento de San Martín no fue incluido). Solo se muestrearon cerdos mayores de cuatro semanas de edad y se excluyeron las provincias con una población menor a 1000 cerdos.

\section{Tamaño de Muestra}

La selección del tamaño de muestra fue obtenida mediante la fórmula para muestreo por conglomerado en dos etapas (Segura y Honhold, 2000). En la primera, se usó la fórmula para proporciones en poblaciones finitas para definir la cantidad de conglomerados con una prevalencia del 15\%, un nivel de confianza de $95 \%$ y un error admisible de $5 \%$, obteniéndose 74 como mínimo, pero al distribuirlos según densidad poblacional de cada provincia se consideraron 195 conglomerados.

En una segunda etapa se determinó la cantidad de cerdos a muestrear dentro de cada conglomerado con una prevalencia de $5.3 \%$, considerando el rango máximo de la prevalencia de VPRRS de $4.3 \pm 1 \%$, determinado por Cruz et al. (2006) en un estudio de crianza porcina de explotación extensiva en Colombia. Posteriormente, se determinó que cada conglomerado estaba conformado por cuatro predios que aporten un promedio 
de seis cerdos cada uno (24 cerdos por conglomerado), bajo un error estándar de proporción menor al $1 \%$. Es así que se colectaron sueros de 4526 cerdos a nivel nacional entre octubre de 2015 y marzo de 2016.

\section{Análisis de Laboratorio}

Los sueros se procesaron en la Unidad Centro de Diagnóstico de Sanidad Animal del SENASA, mediante la prueba de ELISA IDEXX PRRS X3 Ab (IDEXX ${ }^{\circledR}$, EEUU), según las instrucciones del fabricante del kit comercial. Se consideró como positivo un suero con un coeficiente $\mathrm{S} / \mathrm{P}$ de $\geq 0.4$.

\section{Análisis de Datos}

La prevalencia se determinó por departamento y a nivel nacional con sus respectivos intervalos de confianza al $95 \%$. Se evaluaron las variables sexo y edad, estableciéndose cuatro categorías: 1) 4 a 8 semanas (los individuos jóvenes tienen relación con la marrana); 2) 9 a 26 semanas (cerdos confinados en corrales de engorda, lo que aumenta la probabilidad de contagio); 3) 27 a 52 semanas (cerdos seleccionados para reproducción); 4) mayores a 52 semanas (cerdos reproductores con la mayor probabilidad de diseminar el virus).

Los departamentos fueron agrupados de manera arbitraria en zona norte, centro y sur para una mejor interpretación de los resultados. Se utilizó la prueba de Chi-cuadrado de Pearson para establecer asociaciones estadísticas. Los datos fueron analizados en el software estadístico STATA ${ }^{\circledR}$ v. 12.

\section{Resultados}

El $17.3 \%$ de las muestras $\left(\mathrm{IC}_{95 \%}: 16.2-\right.$ $18.5 \%)$ tuvieron anticuerpos contra el VPRRS. Los promedios de la seroprevalencia del VPRRS en los cerdos del norte, centro y sur fueron $14.8 \%\left(\mathrm{IC}_{95 \%}: 12.9-16.7\right), 25.7 \%$ $\left(\mathrm{IC}_{95 \%}: 23.6-27.9\right)$ y $11.5 \%\left(\mathrm{IC}_{95 \%}: 10.0-13.1\right)$, respectivamente. La mayor seroprevalencia del VPRRS fue detectado en las muestras de cerdos de Lima con $62.2 \%\left(\mathrm{IC}_{95 \%}: 57.7\right.$ 66.6), seguido por Arequipa con $37.9 \%$ $\left(\mathrm{IC}_{95 \%}: 31.1-44.6\right)$ y Lambayeque con $31.3 \%$ ( IC $_{95 \%}: 22.0-40.5$ ) (Cuadro 1).

En el Cuadro 2 se presentan los resultados según la edad y sexo. El promedio de la seroprevalencia del VPRRS en hembras fue de $18.7 \%\left(\mathrm{IC}_{95 \%}: 17.3-20.3\right)$ y en machos fue de $15.2 \%$ ( IC $\left._{95 \%}: 13.6-16.9\right)$ $(p<0.05)$. En forma similar, las hembras presentaron una seropositividad mayor que los machos $(\mathrm{p}<0.05)$ en el grupo de cerdos de 27 a 52 semanas de edad.

\section{Discusión}

El $17.3 \%$ de la población cerdos de crianza no tecnificada a nivel nacional con presencia de anticuerpos contra el VPRRS indica que los animales fueron expuestos al VPRRS de campo, ya que el uso de las vacunas comerciales para la prevención del PRRS no está permitido en el país y, por tanto, los cerdos no son vacunados contra esta enfermedad. Los resultados, sin embargo, no permiten precisar el momento en que los animales fueron expuestos. Tampoco existe información sobre la presencia de la enfermedad clínica, sea reportado por los propietarios o por personal del SENASA en las regiones o zonas de procedencia de los cerdos muestreados, ya que los criadores no llevan registros del estado sanitario de sus animales.

El resultado de la infección por el VPRRS puede ser de tipo subclínico o agudo, dependiendo de varios factores, entre ellos, el grado de virulencia de la cepa viral y la presencia de otros patógenos secundarios. No obstante, una buena respuesta inmunitaria humoral es evidente desde la primera semana pos-infección, tanto en el tipo subclínico como en el agudo (Lopez y Osorio, 2004; Amadori y Razzuoli, 2014), lo cual es detectado por la prueba de ELISA. 
Cuadro 1. Seroprevalencia del virus Síndrome Reproductivo y Respiratorio Porcino en cerdos de crianza no tecnificada en 23 departamentos del Perú (2015-2016)

\begin{tabular}{|c|c|c|c|c|c|}
\hline Zona & Departamento & $\begin{array}{c}\text { Muestras } \\
\text { (n) }\end{array}$ & $\begin{array}{c}\text { Muestras } \\
\text { seropositivas } \\
\text { (n) }\end{array}$ & $\begin{array}{c}\text { Prevalencia } \\
(\%)\end{array}$ & IC $95 \%$ \\
\hline \multirow[t]{8}{*}{ Norte } & Tumbes & 72 & 8 & 11.1 & $3.9-18.3$ \\
\hline & Piura & 240 & 62 & 25.8 & $20.3-31.4$ \\
\hline & Lambayeque & 96 & 30 & 31.3 & $22.0-40.5$ \\
\hline & La Libertad & 268 & 40 & 14.9 & $10.7-19.2$ \\
\hline & Cajamarca & 347 & 42 & 12.1 & $8.7-15.5$ \\
\hline & Amazonas & 168 & 10 & 6.0 & $2.4-9.5$ \\
\hline & Loreto & 148 & 6 & 4.1 & $0.9-7.2$ \\
\hline & Subtotal & 1339 & 198 & 14.8 & $12.9-16.7$ \\
\hline \multirow[t]{7}{*}{ Centro } & Ancash & 474 & 19 & 4.0 & $2.2-5.8$ \\
\hline & Lima & 452 & 281 & 62.2 & $57.7-66.6$ \\
\hline & Huánuco & 288 & 35 & 12.2 & 8.4-15.9 \\
\hline & Pasco & 51 & 13 & 25.5 & $13.5-37.4$ \\
\hline & Junín & 222 & 44 & 19.8 & $14.6-25.1$ \\
\hline & Ucayali & 72 & 9 & 12.5 & 4.9-20.1 \\
\hline & Subtotal & 1559 & 401 & 25.7 & $23.6-27.9$ \\
\hline \multirow[t]{11}{*}{ Sur } & Ica & 120 & 6 & 5.0 & $1.1-8.9$ \\
\hline & Huancavelica & 168 & 8 & 4.8 & $1.5-8.0$ \\
\hline & Apurímac & 168 & 10 & 6.0 & $2.4-9.5$ \\
\hline & Ayacucho & 220 & 10 & 4.5 & $1.8-7.3$ \\
\hline & Cusco & 274 & 25 & 9.1 & $5.7-12.5$ \\
\hline & Madre de Dios & 72 & 0 & 0 & 0 \\
\hline & Arequipa & 198 & 75 & 37.9 & $31.1-44.6$ \\
\hline & Moquegua & 72 & 15 & 20.8 & $11.5-30.2$ \\
\hline & Puno & 240 & 19 & 7.9 & $4.5-1.3$ \\
\hline & Tacna & 96 & 20 & 20.8 & $12.7-28.9$ \\
\hline & Subtotal & 1628 & 188 & 11.5 & $10.0-13.1$ \\
\hline Total & & 4526 & 787 & 17.3 & $16.3-18.5$ \\
\hline
\end{tabular}

Los anticuerpos detectados por ELISA son mayormente anticuerpos no neutralizantes inducidos en la proteína $\mathrm{N}$ del PRRSV, que es altamente inmunogénica y abundante (Dea et al., 2000), pero no tienen la capacidad de restringir la infección en el animal permitiendo la circulación del virus en presencia de los anticuerpos no neutralizantes por 150 días o más de la infección. El virus es eventualmente eliminado del organismo animal debido al incremento de los niveles de anticuerpos neutralizantes que se desarrollan lentamente a partir de la segunda semana posinfección (Allende et al., 2000), inducidos principalmente por las glicoproteínas GP5 presentes en la envoltura viral (Wissink et al., 2005; Martínez-Labo et al., 2011). Los anticuerpos neutralizantes son detectados por la prueba de neutralización viral (Kim y Yoong, 2008). 
Cuadro 2. Seroprevalencia del virus del Síndrome Reproductivo y Respiratorio Porcino de cerdos de crianza no tecnificada a nivel nacional, según sexo y edad (2015-2016)

\begin{tabular}{|c|c|c|c|c|c|c|}
\hline \multirow{2}{*}{$\begin{array}{c}\text { Edad } \\
\text { (semanas) }\end{array}$} & \multirow[b]{2}{*}{$\operatorname{Sexo}^{1}$} & \multirow{2}{*}{$\begin{array}{l}\text { Muestras } \\
\text { (n) }\end{array}$} & \multirow{2}{*}{$\begin{array}{c}\text { Muestras } \\
\text { seropositivas } \\
\text { (n) }\end{array}$} & \multirow{2}{*}{$\begin{array}{l}\text { Prevalencia } \\
(\%)\end{array}$} & \multicolumn{2}{|c|}{ IC 95\% } \\
\hline & & & & & Mínimo & Máximo \\
\hline \multirow[t]{2}{*}{$4-8$} & $\mathrm{H}$ & 317 & 49 & $15.4^{\mathrm{a}}$ & 11.4 & 19.4 \\
\hline & M & 279 & 36 & $12.9^{\mathrm{a}}$ & 9.0 & 16.8 \\
\hline \multirow[t]{2}{*}{$9-26$} & $\mathrm{H}$ & 1094 & 171 & $15.5^{\mathrm{a}}$ & 13.4 & 17.7 \\
\hline & M & 830 & 111 & $13.4^{\mathrm{b}}$ & 10.0 & 15.6 \\
\hline \multirow[t]{2}{*}{$27-52$} & $\mathrm{H}$ & 552 & 119 & $21.5^{\mathrm{a}}$ & 18.1 & 25.0 \\
\hline & M & 323 & 51 & $15.8^{\mathrm{b}}$ & 11.8 & 19.8 \\
\hline \multirow[t]{2}{*}{$>52$} & $\mathrm{H}$ & 768 & 175 & $22.8^{\mathrm{a}}$ & 19.8 & 25.8 \\
\hline & $\mathrm{M}$ & 363 & 76 & $20.9^{\mathrm{a}}$ & 16.8 & 25.1 \\
\hline \multirow[t]{2}{*}{ Total } & $\mathrm{H}$ & 2731 & 513 & $18.7^{\mathrm{a}}$ & 17.3 & 20.3 \\
\hline & M & 1795 & 274 & $15.2^{\mathrm{b}}$ & 13.6 & 16.9 \\
\hline
\end{tabular}

${ }^{1} \mathrm{H}$ : hembra; $\mathrm{M}$ : macho

Los anticuerpos contra el VPRRS fueron detectados en cerdos de 22 de los 23 departamentos muestreados (Cuadro 1). Se puede observar que la seroprevalencia del VPRRS es baja en áreas donde no hay mayor desarrollo de la porcicultura tecnificada, como sucede en el trópico y varios departamentos de la sierra sur del país. Se observa también una mayor seroprevalencia en cerdos de áreas cercanas a departamentos costeros donde existe gran cantidad de granjas tecnificadas como Lambayeque, Lima, Arequipa, Moquegua y Tacna, sugiriendo que el VPRRS fue o está siendo difundido desde la costa. La forma más común de difusión del VPRRS en un área, región o país es mediante el transporte de animales vivos, carne cruda o subproductos de origen porcino, semen, y deficiencias en bioseguridad (Mortensen et al., 2002; Sharma et al., 2016). No se tiene información de cómo y cuándo el virus fue introducido a la población de cerdos muestreados, no hubo evidencia clínica de la enfermedad al momento del muestreo, ni tam- poco hubo referencia del incremento de algún problema sanitario por los dueños.

Una de las características del VPRRS es su alta transmisibilidad y facilidad de ser transportado a través de diversas fuentes como animales aparentemente sanos, vehículos y fómites, entre otros (Drew, 2011; Opriessnig et al., 2011) y una vez que el virus ingresa a una granja con alta densidad de cerdos, se difunde rápidamente si no se detecta a tiempo y no se toman medidas de control. La prevalencia e incidencia del VPRRS en granjas tecnificadas es variable y en algunos casos el virus es endémico en los animales de recría y engorda, donde la prevalencia suele llegar hasta el 90\% (Calcina et al., 2014).

Existe escasa información de la prevalencia del VPRRS en cerdos de crianza no tecnificada o familiar en países de América Latina. En un estudio en este tipo de crianza en Colombia se encontró una prevalencia de 
4.3\%, explicada por el control del PRRS en granjas de crianza intensiva (Cruz et al., 2006). En México se reporta una prevalencia de 16.2 y $5.5 \%$ en la región de Ixtlahuaca y Metepec, respectivamente (Milo et al., 2001, citado por Cruz et al., 2006), mientras en República Dominicana se encontró una prevalencia de 1.9\% (Ventura et al., 2013). Por otro lado, Brasil, país con mayor producción porcina, se encuentra aparentemente libre de la infección viral (Ciacci-Zanella et al., 2004). Al parecer, la presencia del VPRRS en la población porcina de crianza no tecnificada es baja debido a una menor densidad poblacional y menor número de cerdos por criador, donde la infección sería autolimitante como ocurre con el pestivirus diarrea viral bovina en pequeños hatos bovinos (Ståhl et al., 2008).

El VPRRS infecta cerdos de todas las edades y sexos, aunque los lechones y gorrinos son los más afectados. La presencia de anticuerpos en los lechones de 4 a 8 semanas de edad podrían ser anticuerpos pasivos o inducidos por el virus de campo. La seroprevalencia fue similar en hembras y machos hasta las 26 semanas de edad; sin embargo, en cerdos de 27 a 52 semanas de edad se encontró una asociación significativa $(p<0.05)$ en cuanto a porcentajes de seropositividad y el sexo de los cerdos (Cuadro 2). Esta asociación podría deberse a la etapa reproductiva en las hembras pues se menciona que la edad y la raza son factores que influencian en la respuesta inmune frente al estrés o agentes infecciosos (Sutherland et al., 2005). Usualmente la prevalencia de un agente infeccioso suele ser mayor en animales adultos por el tiempo de exposición, lo que explicaría el ligero incremento de la seroprevalencia en cerdos mayores a 52 semanas de edad. El presente estudio servirá de base para la vigilancia a través de monitoreos periódicos, así como para evaluar los factores de riesgo y elaborar estrategias preventivas en beneficio de este sector pecuario del país.

\section{Conclusiones}

- El virus del Síndrome Reproductivo y Respiratorio Porcino (VPRRS) está difundido en cerdos de crianza no tecnificada en 22 de los 23 departamentos evaluados del Perú, con una seroprevalencia de $17.4 \%\left(\mathrm{IC}_{95 \%}: 16.2\right.$ $18.5 \%$ ).

- Las muestras provenientes de la zona del centro del país presentaron una seroprevalencia de $25.7 \%\left(\mathrm{IC}_{95 \%}: 23.6\right.$ $27.9 \%$ ), seguido por las muestras de la zona norte con $14.8 \%\left(\mathrm{IC}_{95 \%}: 12.9\right.$ $16.7 \%)$ y del sur con $11.5 \%\left(\mathrm{IC}_{95 \%}: 10.0\right.$ $13.1 \%$ ), donde se concentra la mayor cantidad del ganado porcino de crianzas no tecnificadas a nivel nacional.

- Hubo una asociación estadística $(\mathrm{p}<0.05)$ entre el sexo de los cerdos y la seropositividad al VPRRS dentro del grupo de cerdos entre 27 y 52 semanas de edad.

\section{Literatura Citada}

1. Alegría ME, Rivera H, Manchego A. 1998. Evidencia del virus del Síndrome Reproductivo y Respiratorio Porcino en porcinos de crianza tecnificada. Rev Inv Pec IVITA 9(1): 53-58.

2. Allende $R$, Laegreid $W W$, Kitish GF, Galeota JA, Wills RW, Osorio FA. 2000. Porcine reproductive and respiratory syndrome virus: description of persistence in individual pigs upon experimental infection. J Virol 74: 1083410837. doi: 10.1128/JVI.74.22.1083410837.2000

3. Amadori M, Razuoli E. 2014. Immune control of PRRS: lessons to be learned and possible ways of forward. Front Vet Sci 1(2): 1-14. doi: 10.3389/fvets.2014.00002 
4. Calcina I, Rivera H, Ramírez M, More J, Arroyo G, Acosta F, Manchego A. 2014. Cinética de anticuerpos contra el virus del síndrome reproductivo y respiratorio porcino durante las etapas de recría, engorde y acabado en una granja de Lima. Rev Inv Vet Perú 25: 88-94. doi: 10.15381/rivep.v25i1.8472

5. Ciacci-Zanella JR, Trombetta C, Vargas I, Mariana da Costa DE. 2004. Lack of evidence of porcine reproductive and respiratory syndrome (PRRSV) infection in domestic swine in Brazil. Cienc Rural 34: 449-455. doi: 10.1590/S0103-84782004000200018

6. Cruz MC, Mogollón JD, Rincón MA, Peña NB, RuizS, Lora AM. 2006. Prevalencia serológica del Síndrome Reproductivo y Respiratorio Porcino (PRRS) en cerdos de explotaciones extensivas de Colombia. Rev Med Vet Zoot 53: 33-41. doi: 10.15446/rfmvZ

7. Dea S, Gagnon CA, Mardassi $H$, Pirzadeh B, Rogan D. 2000. Current knowledge on the structural proteins of porcine reproductive and respiratory syndrome (PRRS) virus: comparison of the North American and European isolates. Arch Virol 145: 659-688. doi: 10.1007/s007050050662

8. Drew TW. 2011. The emergence and evolution of swine viral diseases: to what extent have husbandry systems and global trade contributed to their distribution and diversity? Rev Sci Tech 30: 95-106. doi: 10.20506/rst.30.1.2020

9. Holtkamp D, Kliebenstein J, Neumann E, Zimmerman J, Rotto H, Yoder T, Wang P, et al. 2013. Assessment of the economic impact of porcine reproductive and respiratory syndrome virus on United States pork producers. J Swine Health Prod 21: 72-84.

10. [INEI] Instituto Nacional de Estadística e Informática. 2012. IV Censo agropecuario 2012. [Internet]. Disponible en: http://proyectos.inei.gob.pe/ w e b / D o cum entos Publicos / ResultadosFinalesIVCENAGRO.pdf
11. Kim WI, Yoong KJ. 2008. Molecular assessment of the role of envelopeassociated structural proteins in cross neutralization among different PRRS viruses. Virus Genes 37: 380.391. doi: 10.1007/s11262-008-0278-1

12. Lambert ME, Arsenault J, Poljak Z, D'Allaire S. 2012. Epidemiological investigations in regard to porcine reproductive and respiratory syndrome (PRRS) in Quebec, Canada. Part 2: prevalence and risk factors in breeding sites. Prev Vet Med 104: 84-93. doi: 10.1016/j.prevetmed.2011.11.002

13. Lopez OJ, Osorio FA. 2004. Role of neutralizing antibodies in PRRS protective immunity. Vet Immunol Immunopathol 102: 155-163. doi: 10.1016/j.vetimm.2004.09.005

14. Martinez-Labo FJ, Diez-Fuertes F, Simarro I, Castro JM, Prieto C. 2011. Porcine reproductive and respiratory virus isolates differ in their susceptibility to neutralization. Vaccine 29: 6928-6940. doi: 10.1016/j.vaccine.2011.07.076

15. Mortensen S, Stryhn H, Sogaard R, Boklund A, Stärk KD, Christensen J, Willeberg P. 2002. Risk factors for intection of sow herds with porcine reproductive and respiratory (PRRS) virus. Prev Vet Med 53: 83-101. doi: 10.1016/S0167-5877(01)00260-4

16. Morales R, Rebatta M, Lucas J, Mateo J, Ramos D. 2014. Caracterización de la crianza no tecnificada de cerdos en el parque porcino del distrito de Villa el Salvador, Lima-Perú. Salud Tecnol Vet 2: 39-48. doi: 10.20453/ stv.v2i1.2206

17. Opriessing T, Giménez-Lirola LC, Halbur PC. 2011. Polymicrobial respiratory disease in pig. Anim Health Res Rev 12: 133-148. doi: 10.1017/ S1466252311000120

18. [OIE] Organización Mundial de Sanidad Animal. 2004. Manual de la OIE sobre animales terrestres. Capítulo 2.6.5. Síndrome reproductivo y respiratorio porcino. p 862-876. [Internet]. Available in: http://www.oie.int/es/normas-internacionales/codigo-terrestre/ 
19. Ramírez M, Rivera H, Manchego A, More J, Chiok KL. 2013. Aislamiento y genotipificación del virus del síndrome respiratorio y reproductivo porcino (VPRRS) en granjas seropositivas de las provincias de Lima y Arequipa, Perú. Rev Inv Vet Perú 24: 222-232. doi: 10.15381/ rivep.v24i2.2512

20. Segura JC, Honhold N. 2000. Métodos de muestreo para la producción y la salud animal. Yucatán, México: Univ. Autónoma de Yucatán. $160 \mathrm{p}$.

21. Snijder EJ, Kikkert M, Fang Y. 2013. Arterivirus molecular biology and pathogenesis. J Gen Virol 94: 2141-2163. doi: 10.1099/vir.0.056341-0

22. Sharma BK, Manandhar S, Devleesschauwer B. 2016. Serological evidence of type 2 (North American genotype) porcine reproductive and respiratory syndrome virus in Nepal. Trop Anim Health Prod 48: 663-666. doi: 10.1007/s11250-015-0986-1

23. Ståhl K, Lindberg A, Rivera H, Ortiz C, Moreno-López J. 2008. Selfclearance from BVDV infections - a frequent finding in dairy herds in an endemically infected region in Peru. Prev Vet Med 83: 285-296. doi: 10.1016/ j.prevetmed.2007.08.005
24. Sutherland MA, Rodriguez-Zas SL, Ellis M, Salak-Johnson JL. 2005. Breed and age affect baseline immune traits, cortisol and performance in growing pigs. J Anim Sci 83: 2087-2095. doi: $10.2527 / 2005.8392087 \mathrm{x}$

25. Wissink EH, Kroese MU, vanWijk HA, Rijsewijk FA, Meulenberg JJ, Rottier PJ. 2005. Envelope protein requirements for the assembly of infectious virions of porcine reproductive and respiratory syndrome virus. J Virol 79: 12495-12506. doi: 10.1128/ JVI.79.19.12495-12506.2005

26. Ventura A, González W, Barrette R, Swenson S, Bracht A, Rowland J, Fabian A, et al. 2013. Virus and antibody diagnostics for swine samples of the Dominican Republic collected in regions near the border to Haiti. ISRN Virology 2013: ID 425831. doi: 10.5402/ 2013/425831

27. Zimmerman J, Yoon KJ, Wills RW, Swenson RL. 1997. General overview of PRRSV: a perspective from the United States. Vet Microbiol 55: 187-196. doi: 10.1016/S0378-1135(96)01330-2 